\title{
New Weapon of Tomorrow's Battlefield Driven by Hypersonic Velocity
}

\author{
Bahman Zohuri ${ }^{1}$, Patrick McDaniel ${ }^{2}$, Jim Lee ${ }^{3}$ and Casey John Rodgers ${ }^{4}$ \\ 1. Electrical and Computer Engineering Department, University of New Mexico, Albuquerque, New Mexico 87131, United States \\ 2. Nuclear Engineering Department, University of New Mexico, Albuquerque, New Mexico 87131, United States \\ 3. LANL/SNL, Manager (Retired), Edge Wood New Mexico 87015, United States \\ 4. Electrical and Computer Engineering Department, University of New Mexico, Albuquerque, New Mexico 87131, United States
}

\begin{abstract}
Speed is the new stealth and earlier this week America's top nuclear commander described a grim scenario for U.S. forces facing off against hypersonic weapons. "We do not have any defense that could deny the employment of such a weapon against us," Air Force Gen. John Hyten, commander of U.S. Strategic Command, told the Senate Armed Services Committee on Tuesday March 20 , 2018. Russian and Chinese are aggressively developing new weapons that travel at Mach 5 or higher, which is at least five times faster than speed of sound (hypersonic). These weapons travel in excess of 3,600 miles per hour (1 mile per second) and currently, no military possesses a credible defense. Finding, tracking and intercepting something that fast is unprecedented. Given that Russia and China have invested heavily in advanced defensive technologies that now hold most of our traditional forms of power projection at risk, this is a significant advantage - it is one that would impose major costs upon a defending nation. Recently, according to the director of the Army’s Rapid Capabilities and Critical Technologies Office (ARCCTO), The Army will field a battery of truck-borne hypersonic missiles in 2023, with a contract award in August, the service's new three-star Program Executive Officer said. The service will also field a battery of 50-kilowatt lasers on Stryker armored vehicles by 2021, he said. A program to put a 100-plus-kilowatt laser on a heavy truck, however, is under review and may be combined with Air Force and/or Navy efforts to reach comparable power levels, Lt. Gen. Neil Thurgood told reporters in his interview. In this white paper we are suggesting a new technology as a counter-measure against such an adversary measure and threat that is aggressively being pursued by these two nations, Russia and China both tactically and strategically. We also briefly discuss possible physics and science of aerodynamics involved with these vehicles traveling between range of 5 Mach and higher, where we discuss current status and future direction driven by phenomena of plasma aerodynamics thorough possibly, weakly ionized gases (WIG) program that was started by the former Soviet Republics under AJAX Vehicle and that was direct understanding of the role of plasmas in the performance of this vehicle.
\end{abstract}

Key words: Hypersonic velocity, hypersonic flying object, weak plasma gas, high power microwave, air breakdown, hypersonic weapons.

\section{Introduction}

On December 26, 2018, Russia successfully carried out the launch of a liquid-fueled intercontinental-range ballistic missile (ICBM) carrying the Avangard hypersonic glide vehicle payload. The Avangard is a modernized Russian delivery vehicle, designed to maneuver in the upper atmosphere at speeds in excess of Mach 5. See the link below on YouTube and the artistic image depicted in Fig. 1.

Corresponding author: Bahman Zohuri, Ph.D., associate professor, research fields: electromagnetic and plasma physics.
The missile that carries the Avangard hypersonic glide vehicle is the UR-100 NUUTKh, an ICBM-class missile. As the diplomat reported earlier this year, Russian defense industry sources had noted that the first Russian Strategic Missile Forces regiment to operate the Avangard would oversee a test later this year [1].

"The launch was performed by an operational unit of the Strategic Missile Forces from Dombarovsky missile deployment area against a hypothetical target at the Kura range, Kamchatka Territory,” the Kremlin noted in a statement. 


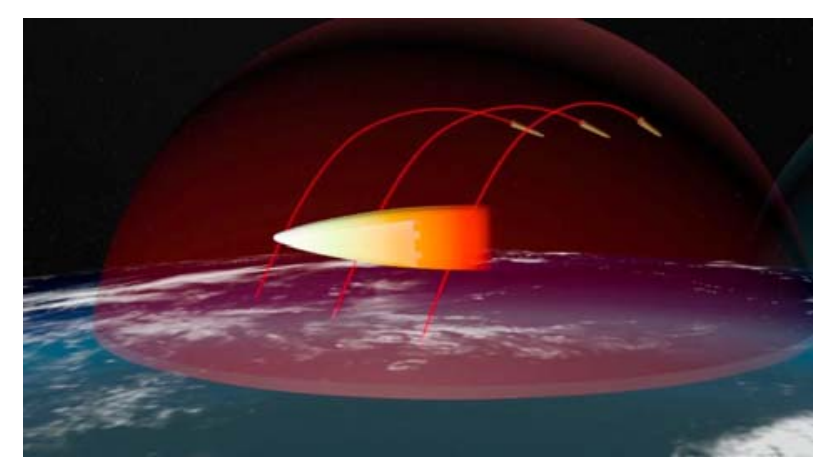

Fig. 1 Artistic depiction of glide hypersonic warhead weapon.

Source: https://www.youtube.com/watch?v=tKa31NaYsNw.

"Flying at hypersonic speed, the glide vehicle performed vertical and horizontal maneuvers and hit the hypothetical target in time within the range's combat field," the statement added [1].

Footage of the successful test was released immediately by Russian media, showing the missile's hot launch from a silo. The Avangard glider itself was not seen in any released footage. The missile's payload separates after the booster's powered flight into the exoatmosphere. After the resulting ballistic trajectory, the hypersonic glide vehicle descends and maneuvers in the upper atmosphere on its way to the target.

Putin emphasized the Avangard's primary purpose. "The new Avangard system is impervious to current and future air defense and missile defense systems of a potential enemy," he noted [1].

Hypersonic missiles developed by China and Russia are being used to justify the reprise of space-based missile defense systems by the Pentagon.

The U.S. is particularly concerned about super-fast guided missiles under development in China that could put US ships and bases at risk in Asia. China's DF-26 ballistic missile drill sent a clear message to the US (see Fig. 2).

In the case of the Russian hypersonic weapon Avangard, according to them, it maneuvers to bypass missile defenses enroute to the target. President Vladimir Putin has declared that Russia has developed a range of new nuclear weapons, which cannot be intercepted by an enemy.

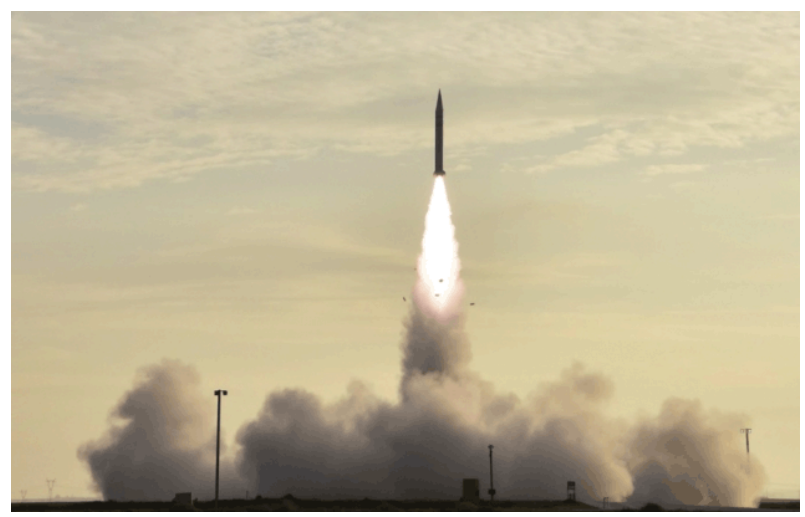

Fig. 2 Chinese DF-26 launch.

Machine guns, fighter jets, nuclear weapons, when a new facet of military technology gains operational capability, sometimes it changes the rules of the game. Hypersonic weapons - that travel over five times the speed of sound-are difficult to detect and harder to intercept, offer that kind of potential.

\section{The History of Hypersonic Vehicle}

In 1994, Russian physicists and scientists were claiming and introduced an innovative and novel hypersonic flight vehicle concept that is known to us as AJAX or AYAKS through public domain articles and literatures. AJAX was described as a scramjet-powered vehicle driven by plasma-based technology with two purposes behind it as:

(1) combustion, and;

(2) aerodynamics performance.

Each of the above ideas has their own scientific ideology behind the purpose incorporating physics of weakly ionized gases (WIG) according to US Air Force Research Laboratory (AFRL) and the European Office of Aerospace Research and Development (EOARD) in response to the Russian AJAX program. The WIG program was established to head on and foster US-Eastern Bloc in order to collaborate and exchange on the recent interest on the subject of "plasma aerodynamics” science.

This field of science led to an international study and collaboration, which was stimulated by reveal and disclosure of the Soviet AJAX vehicle concept in the 
mid-1990s and true belief today in Russia and China in respect to what hypersonic weapons are is the dove tail of AJAX program started by former Soviet Union around 1990s time frame.

Plasma-based flow control seems very feasible, in particular for local flow control applications where power consumption is low, where new methods utilize magneto-hydro-dynamic (MHD), flow dynamic and electro-dynamic are couple to form MHD technique, which already led to and it is employed in order to control and power extraction is already leading to innovative designs for hypersonic vehicles to address both issues of combustion and aerodynamic performance by increasing speed while reducing drag and friction like we can see in case of re-entry vehicle.

Keep in your mind that the fundamental equations of "Plasma Dynamics" have been greatly simplified by MHD approximation, which uses the assumptions given and summarized below [2]:

(1) Plasma is a single continuous medium of definite composition.

(2) Electromagnetic forces are of the same order as gas dynamic forces.

(3) The time scale of the problem is the characteristic length divided by characteristic velocity.

(4) The applied electric field $\vec{E}$ is of the same order as the induced electromotive force.

(5) The flow velocity is much smaller than the speed of light.

(6) Maxwell's equations are unaffected by gas dynamic motion or, in other words, the magnetic field $\vec{B}$ induced by the fluid motion is small compared to the applied magnetic field.

(7) Inviscid flow is assumed, but a friction term can be easily added to the momentum equation.

(8) No heat loss is assumed, but a heat loss term can be easily added to the energy equation.

(9) The equation of state of the gas is assumed to be the perfect gas law, but other equations of state can be implemented.

(10) Body forces due to gravity are neglected.
(11) Steady-state is assumed.

All these above points can be simplified in generalized 3-D magnetohydrodynamic (MHD) equations written as follows [3]:

Momentum equation (Eq. (1)):

$$
\rho(\overrightarrow{\mathrm{v}} \cdot \vec{\nabla}) \overrightarrow{\mathrm{v}}=\vec{J} \times \vec{B}-\nabla p
$$

Mass equation (Eq. (2)):

$$
\vec{\nabla} \cdot(\rho \overrightarrow{\mathrm{v}})=0
$$

Energy equation (Eq. (3)):

$$
\rho \overrightarrow{\mathrm{v}} \cdot \vec{\nabla}\left(\frac{|\overrightarrow{\mathrm{v}}|^{2}}{2}+U\right)=-\vec{\nabla} \cdot(\overrightarrow{\mathrm{v}} p)+\vec{J} \cdot \vec{E}
$$

Current equation (Eq. (4)):

$$
\vec{\nabla} \cdot \vec{J}=0
$$

Ohm's law (Eq. (5)):

$$
\vec{J}=\sigma(\vec{E}+\overrightarrow{\mathrm{v}} \times \vec{B})-\frac{\omega \tau}{|\vec{B}|} \vec{J} \times \vec{B}
$$

In above sets of conservation equations, the following nomenclatures apply:

$\vec{B}$ : Vector magnetic field

$\vec{J}$ : Vector current density

$\rho:$ Gas density

$\overrightarrow{\mathrm{v}}$ : Vector velocity of flow

$U$ : Internal energy of gas

$p$ : Flow pressure

$\vec{E}$ : Vector of applied electric field

$\omega \tau$ : Hall parameter

$\sigma$ : Electric conductivity

The Russian AJAX hypersonic vehicle was the first to propose MHD energy bypass of a scramjet as a means of extending the scramjet's performance to higher Mach numbers [4].

Further analyses of the MHD concept lead to the conclusion that energy bypass of a scramjet can result in subsonic ramjet propulsion which can supersede and be able to maintain speed in the range of Mach number 
between 10 and 16 [5].

A simplified thermodynamic cycle analysis of scramjet energy bypass demonstrated that the concept merits further investigation [6]. Based on these results, an examination of the feasibility of MHD energy bypass with turbojets was proposed [7].

As with the scramjet, the enthalpy into the combustor is to be reduced allowing more efficient addition of energy in the combustor without exceeding temperature limitations on the turbine materials. Preliminary 1-D analysis of the energy extraction process shows that significant enthalpy extraction is possible, but this extraction also results in significant total pressure losses [8].

Today, plasma enhanced combustion is behind $85 \%$ of primary energy conversion processes that are based on combustion, and this proportion is expected to remain stable in the foreseeable future. However, AJAX project triggered interest in the use of plasma discharges as a means to provide in-place, on-demand enhancement of fuel-air reactivity, and thus launched the new field of plasma-assisted combustion.

To continue our discussion with flight control and reduced surface heating can also be achieved through the use of magnetic field interaction with the bow shock as illustrated in Fig. 3 in respect to the other shock as illustrated in Fig. 4, and concept is currently under AFRL supervision research program for implementation of some kind of flight test conceptual study.

And conceptual image of the other three main types of shock waves, namely:

(1) Normal shock wave;

(2) Oblique shock wave, and;

(3) Bow shock wave.

Note that, while "Normal Shock" is a normal shock wave occurs completely parallel to the surface as shown in Fig. 4. Furthermore, the oblique shock wave is usually at angle and the bow shock wave is completely parabolic. The shape of the nose is designed in order to generally create a bow shock (usually circular nose).

The "Bow Shock" wave forms, when the aircraft is flying at a speed faster than speed of sound (i.e. Mach number $=1$ ). A bow wave is a shock wave in front of a body, such as an airfoil, or is apparently attached to the forward tip of the body as it is depicted in Fig. 5.

The concept of reducing surface heating and consequently drag/friction driven by with assist from magnetic field interacting with bow shock as it was explained in preceding paragraph under continues industrial investigation (i.e. implementation of Wingtip Canard), as demonstrated in Fig. 6.

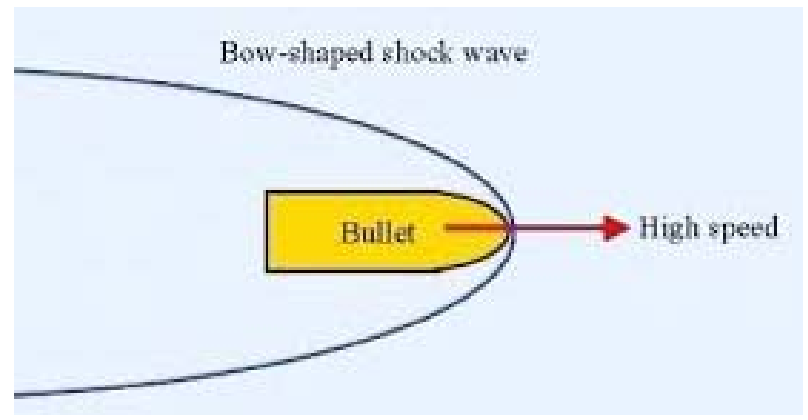

Fig. 3 Bow-shaped shock wave.

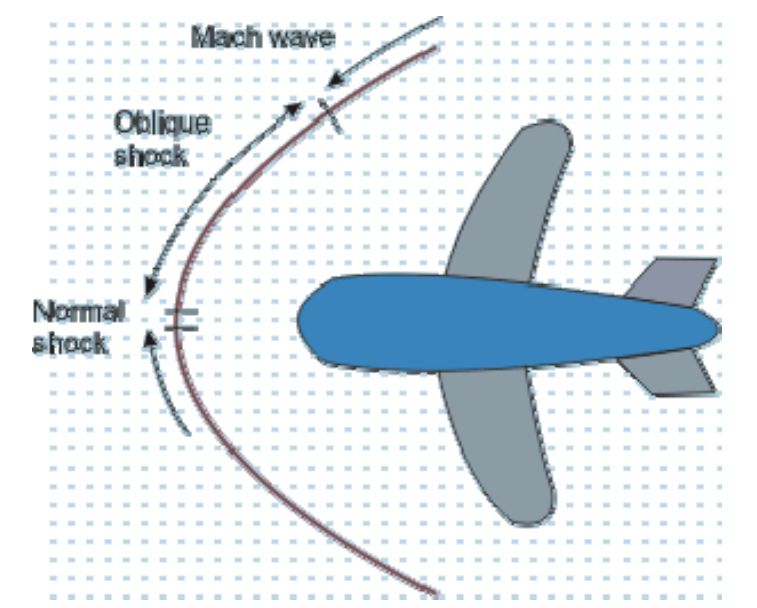

Fig. 4 All three-shock waves presentation.

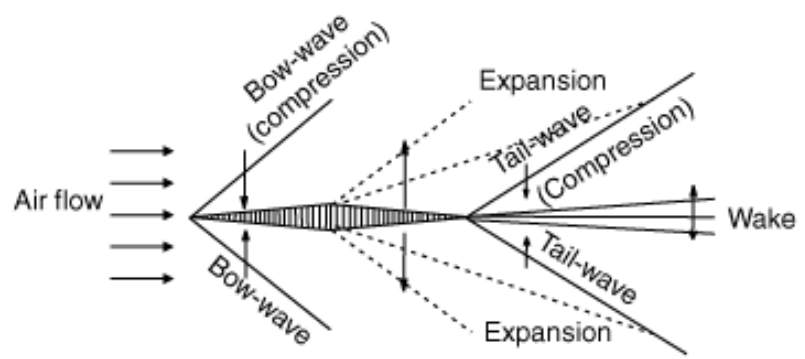

Fig. 5 Bow shock wave illustration. 


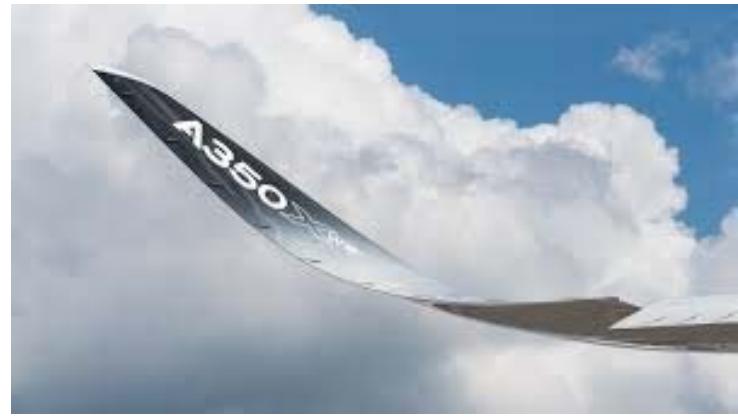

Fig. 6 Wingtip Canard surface.

Wingtip devices such as "Canard" are intended to improve the efficiency of fixed-wing aircraft by reducing drag.

Industry seems to be receptive to the adoption of plasma-based technology, but has reservations about technical risk, performance, reliability, and integration. There is a need to identify applications where plasma devices are significantly better than completing technologies, and to demonstrate prototypes in an operational environment.

In conclusion, in order to satisfy the two mentioned purposes behind the physics and science of WIG, there are some suggested breakthrough areas suggested by scientist in order to take advantages of plasma-assisted combustion to increase speed of flying vehicle beyond Much 5, a range or higher that hypersonic weapons like to travel either in glide or cruse mode (see Section 2.3 of this white paper), with additional assist from effectiveness of surface plasma actuator, plasma-enhanced aerodynamics, and measurement technology that are expected.

Furthermore, effectiveness of surface plasma actuator is anticipated with new electrode configurations, optimized dielectrics, and optimized high voltage driving waveforms. In addition, breakthroughs are expected to occur with multiple-electrode configurations for thrust generation and shock wave focusing, and further breakthrough may also arise from new surface materials and their characteristics that allow changes in fundamental structure or the temporal evolution of the discharge.

More surface-based concepts can make use of plasma arrays that are capable of generating shock waves that propagate away from the surface and coalesce to generate vorticity or drive acoustic waves for control of near-surface flows, while plasma generated far-ultraviolet radiation may also be taken under consideration for rapid near-surface additional energy driven by direct absorption and molecular dissociation of chemical element in atmosphere such as oxygen.

Breakthrough technology in the measurement area will be enabled via the development of new devices that are capable of interacting with air and combustion environments in ways that are not feasibly practical today. However, maybe in near future such feasibility will be created through rapid progress in direction of developing an efficient and very short-pulsed, yet precision along with controllable, high-power, high-repetition rate lasers. These lasers will open up new methods for real time data acquisition as well as off-body energy addition, efficient volume ionization method for MHD applications, and volumetric, selective radial production for combustion reaction and ignition control. With progress in laser technology and goal toward its miniaturization of new generation of laser at higher efficiency operation in flight will be a practical fact.

Additionally, inspired by the National Aero-Space Program, which started in 1995 in the United Sates, research on hypersonic flight has been actively pursued in various nations. The hypersonic research has been aimed mainly at the development of a reasonable hypersonic vehicle with a supersonic combustion ramjet or SCRAMJET.

One of the most challenging concepts of this type of vehicle is the single stage to orbit vehicle or SSTO vehicle which would take off horizontally and fly up to orbital speeds in the atmosphere. A trans-atmospheric air-breathing vehicle, which needs to provide sufficient air for the engine operation, must be accelerated at relatively lower altitudes compared with the conventional rocket boosters. On the other hand, in 
order to avoid excessive dynamic pressure and aerodynamic heating for the structures and materials, higher altitudes are desirable.

Also bear in mind one of the important aspect object flying at hypersonic speed is creation of plasma sheath around the vehicle during flight time period. From science of plasma physics point of view, when a vehicle flying in the atmosphere at high velocities becomes surrounded by regions of ionized gas that affect the propagation of electromagnetic waves to and from the vehicle. The kinetic energy in a hypersonic free stream is converted to the internal energy of the gas across the strong bow shock wave, creating very high temperatures in the shock layer near the nose. If the temperature is high enough, ionization is present, and a large number of free electrons are produced throughout the shock layer.

Downstream of the nose region, a boundary layer grows along the surface of the vehicle. Since the Mach number at the outer edge of the boundary layer is still high, the intense frictional dissipation within the hypersonic boundary layer creates high temperatures and causes chemical reactions.

The ions and electrons produced in the high temperature air around the vehicle create a plasma sheath, which interacts with electromagnetic waves propagating to and from the vehicle. If the attenuation of the electromagnetic waves due to the plasma sheath is excessively high, then a communication blackout occurs.

However, because of these constraints, the trajectory of a hypersonic vehicle will be constrained into a very narrow region. An important question which should be taken into account for the trajectory is the interference between the electromagnetic waves used for communication and the plasma sheath around the vehicle. This is an important event that needs to be dealt with when it comes to detect an incoming hypersonic weapon at far-field or distance using a high power microwave (HPW) either as a weapon of countermeasure against such measure or as we said yet to detect it, beside obstacle within geodesic distance the electromagnetic wave of this device in transverse electro-magnetic (TEM) mode to go back and forth from source to target and back to source.

However, to overcome this matter, we suggest application of a new family of waves known as scalar longitudinal wave (SLW). It is discussed by Zohuri [9, 10] and it is briefly explained in Section 7 of this white paper.

\subsection{Weakly Ionized Plasmas via MHD and Electrohydrodynamic Controlling Hypersonic Flows}

Theoretical analysis and fundamental aspects of high-speed flow control using electric and magnetic field applying MHD are well understood and established by the scientists [8].

There is a growing interest in using WIG (plasmas) and electric and magnetic fields in high-speed aerodynamics. Wave and viscous drag reduction, thrust vectoring, reduction of heat fluxes, sonic boom mitigation, boundary-layer and turbulent transition control, flow turning, and compression, onboard power generation, and scramjet inlet control are among plasma and MHD technologies that can potentially enhance performance and significantly change the design of supersonic and hypersonic vehicles [8].

Meanwhile, despite many studies devoted to these new technologies, a number of fundamental issues have not been adequately addressed. Any plasma created in gas flow and interacting with electric and magnetic fields would result in gas heating. This heating can certainly have an effect on the flow and, in some cases, can be used advantageously. However, a more challenging issue is whether significant nonthermal effects of plasma interaction with electric and magnetic fields can be used for high-speed flow control.

In conventional MHD of highly conducting fluid, electric and magnetic effects give rise to ponderomotive force terms $\nabla\left(\varepsilon_{0} E^{2} / 2\right)$ and $\nabla\left(B^{2} / 2 \mu_{0}\right)$, which can be interpreted as gradients of electric and magnetic field pressures. These ponderomotive forces are 
successfully utilized for plasma containment in fusion devices and also play an important role in astrophysics. One might hope that these forces can also be used for control of high-speed flow of ionized air. However, the great importance of ponderomotive forces in fusion and astrophysical plasmas is due to the fact that those plasmas are fully, or almost fully, ionized and, therefore, are highly conductive. In contrast, high-speed air encountered in aerodynamics is not naturally ionized, even in boundary layers and behind shocks if the flight Mach number is below about 12, due to the low static temperature. Therefore, ionization has to be created artificially, using various electric discharges or high-energy particle beams [11]. In most conditions, the artificially created plasmas are weakly ionized, with ionization fraction ranging from $10^{-8}$ to $10^{-5}$. Because of the low ionization fraction and electrical conductivity, interaction of the plasma with electromagnetic fields and transfer of momentum and energy to or from the bulk neutral gas can be quite inefficient. Further information can be found in Ref. [8].

In conclusion, the principal difficulty in high speed flow with hypersonic regime flow control using electric and magnetic fields is that the relatively cold gas has to be ionized in electric discharge or by electron beams, which requires large power inputs and results in low ionization fraction and electrical conductivity. The low ionization fraction means that, although electrons and ions can interact with electromagnetic fields, transfer of momentum and energy to or from the bulk neutral gas can be small compared with momentum and energy carried by the high-speed flow.

\subsection{What Is a Hypersonic Weapon?}

A hypersonic weapon is a missile that travels at Mach 5 or higher, which is at least five times faster than the speed of sound. This means that a hypersonic weapon can travel about one mile per second. For reference, commercial airliners fly sub-sonically, just below Mach 1 whereas modern fighter jets can travel supersonically at Mach 2 or Mach 3.

\subsection{What Types of Hypersonic Weapons Are in Development?}

There are two types of weapons emerging (see Fig. 7):

(1) hypersonic cruise missiles and,

(2) hypersonic glide vehicles.

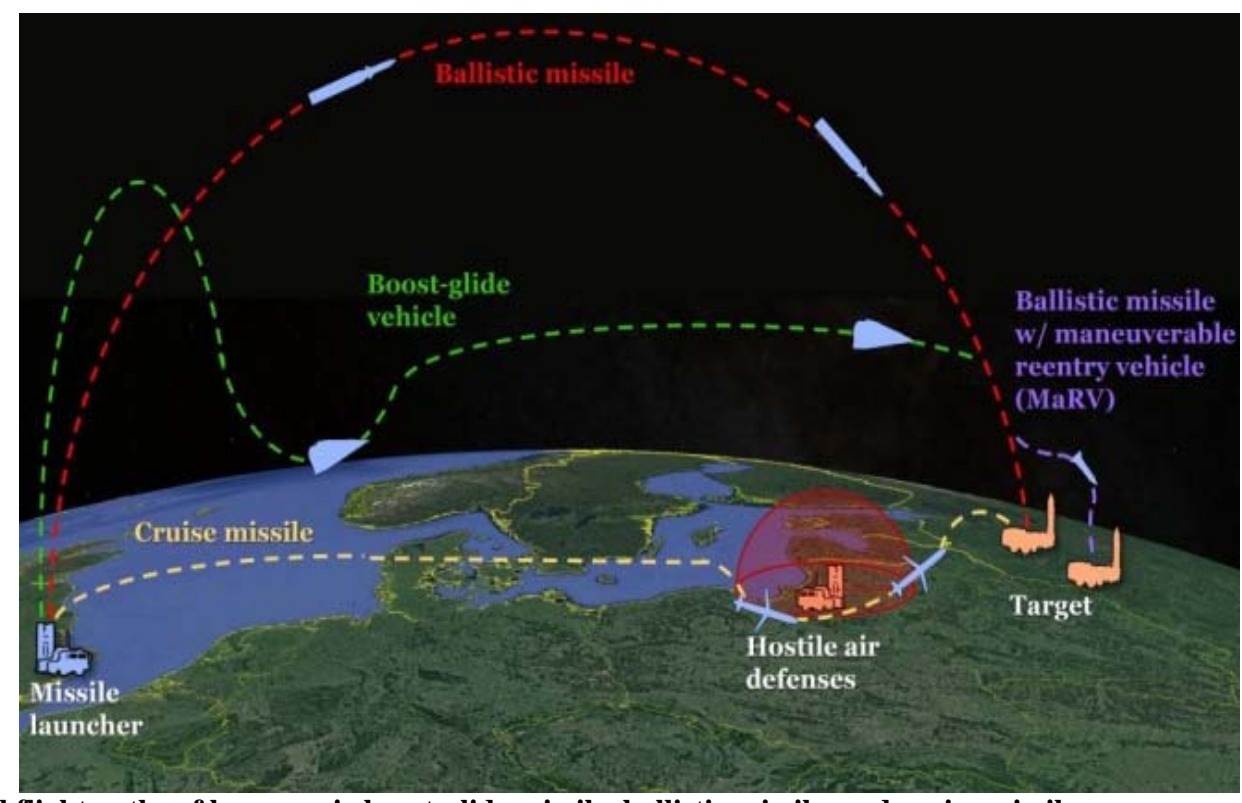

Fig. 7 Notional flight paths of hypersonic boost-glide missile, ballistic missile, and cruise missile.

Source: courtesy of CSBA graphic. 


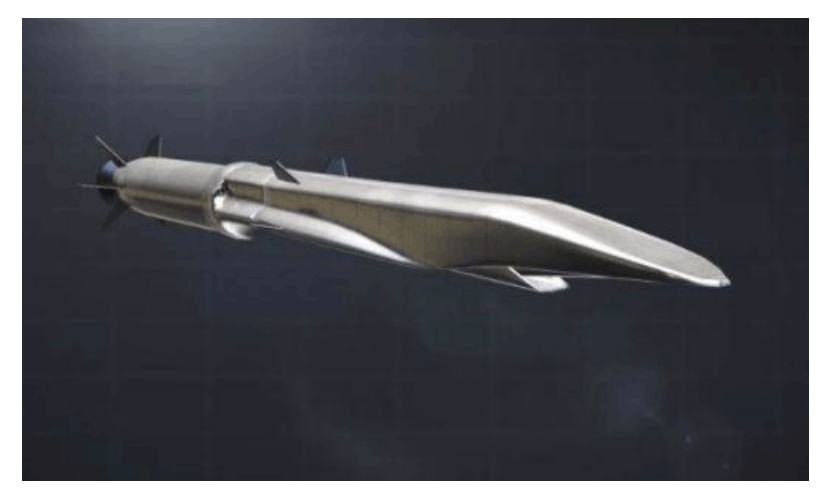

Fig. 8 Cruise type hypersonic weapon.

Hypersonic cruise missiles are powered all the way to their targets using an advanced propulsion system called an SCRAMJET. These are very, very fast. You may have as little as 5 to 20 minutes from the time it launched until the time it strikes, for anticipated standoff ranges (see Fig. 8).

Hypersonic cruise missiles can fly at altitudes up to 100,000 feet whereas hypersonic glide vehicles can fly above 100,000 feet.

Hypersonic glide vehicles are placed on top of rockets, launched, and then glide in the upper atmosphere.

Both are like a plane with no engine. They use aerodynamic forces to maintain stability, to fly along, and to maneuver as well. Furthermore, because they are maneuverable, they can keep their targets a secret up until the last few seconds of their flights.

\subsection{What Are Some Technical Requirements Needed for Hypersonic Weapons?}

Once they reach Mach 5 in flight mode within the atmosphere, they cannot use traditional jet engines to make such vehicles go faster.

They need a completely different design to unclutter the flow path and sustain combustion of the supersonic airflow inside the engine.

The answer becomes a supersonic combustion ramjet (SCRAMJET), which can operate between Mach 5 and Mach 15.

In order to maintain sustained hypersonic flight, a vehicle must also endure the extreme temperatures of flying at such speeds. The air impinging on the frontal surfaces of the vehicles becomes plasma.

One can think of it as flying into a blowtorch. It is very similar circumstance to the return of reentry capsules coming back from orbiting the moon, traveling with or without astronauts in them. The faster a vehicle flies, the pressure and temperature rise exponentially. Thus, they need to have materials that can withstand high temperature over a long period of time during the journey of such a weapon to the designated target.

In conclusion, as we have discussed for hypersonic vehicle to be able to travel at speed above Mach 5 and higher, these types of vehicles need to travel an environment very close to plasma condition either ahead of object or be surrounded by plasma such as plasma sheath that is created either by means of weak ionized gas schema or shock wave ahead of the object, or even some plasma actuator. Thus disturbing such an environment around the vehicle, one can interrupt the pattern of flight trajectory via longitudinal scalar wave (LSW) or some other means, since TEM wave is not able to penetrate the plasma sheath.

\section{Which Countries Are Developing Hypersonic Weapons?}

"The U.S., Russia and China are ahead of other nations in developing hypersonic weapons," Richard Speier, adjunct staff with Rand, told Consumer News and Business Channel (CNBC).

Speier, who worked to initiate the Pentagon's Office of Counter-Proliferation Policy, added that France, India, and Australia are also developing military uses of hypersonic technology.

"Japan and various European countries such as France recently are working on civilian uses of the technology, such as space launch vehicles, or civilian airliners, but civilian uses can be adapted for military purposes," Speier noted.

It is very commonly asserted that there is an arms race in hypersonic technology and that the United 
States is losing. There is certainly an arms race, but it is not obvious or clear and we are not convinced the United States is losing. Experts often argue the United States is behind in this technology because Russia and China appear to be testing more frequently. This is true, but in many ways, the United States is running a different race from Russia and China.

Russia and China appear to be focused primarily on the delivery of nuclear warheads, and in this case, accuracy can be relaxed. The United States is interested in the delivery of non-nuclear warheads, and here, accuracy is absolutely critical for the weapon to be militarily effective. The United States wants to be landing weapons within a few meters of the target with enhanced circular error probable (CEP). U.S. goals are much more demanding than Russian and Chinese goals.

The United States also has a very long history of testing in this area, which gives the United States an advantage in its current efforts. For instance, the most successful U.S. boost-glide weapon R\&D program, the advanced hypersonic weapon, has seen a glider tested over about 4,000 kilometers. China, by contrast, appears to have been testing boost-glide weapons at a range of less than 2,000 kilometers.

So, to summarize, there are two considerations here: the U.S. history in this area, and the inherently more demanding technology that the United States is pursuing. When you take those factors into account, it is easy to conclude the United States is not behind in this competition.

However, the development of hypersonic weapons in the United States, has been largely motivated by technology, not by strategy.

In other words, technologists have decided to try and develop hypersonic weapons because it seems like they should be useful for something, not because there is a clearly defined mission need for them to fulfill. The first-order task for the Department of Defense (DOD) is, therefore, to decide what missions it has that warrant hypersonic weapons. Then we can have a conversation about what the most cost-effective way of achieving those goals is. Is it indeed hypersonic weapons, or is there a better alternative? The real priority task here is for the DOD to develop a strategy for the acquisition of hypersonic weapons, and that is not possible until it is decided what these weapons might be used for.

\section{What Is the Significance of Russia's Latest Missile Efforts?}

In his speech at the beginning of March, President Vladimir Putin presented an extraordinary list of new weapons that he claims Russia is developing or has deployed. This list included a number of hypersonic capabilities [12].

The most significant is a boost-glide weapon called Avangard. This maneuvering weapon, according to Putin, has been designed to defeat U.S. missile defenses. Since Putin's speech, Russia has indicated that the Avangard glider is going to be deployed on at least two different kinds of ballistic missiles and will carry nuclear warheads. It is possible that this weapon could, in the future, be used for the delivery of non-nuclear warheads, if its accuracy can be refined. But in the short term, its only purpose appears to be the delivery of nuclear warheads.

Second, Putin announced a novel boost-glide weapon, called Kinzhal, which means "dagger" in Russian. This weapon is launched from an aircraft and has a shorter range than Avangard. The media reporting we have seen coming out of Russia suggests that this weapon is also nuclear armed. Perhaps counterintuitively, however, the development of nuclear-armed boost-glide vehicles by Russia should be less worrying to us than the development of non-nuclear boost-glide vehicles. Russia already has the capability to deliver nuclear weapons to U.S. and allied targets—and, frankly, we cannot totally deny that capability.

Russian nuclear-armed boost-glide vehicles do not, therefore, change the status quo. If, however, Russia developed boost-glide weapons with non-nuclear 
warheads, it would present a new and potentially very significant security threat to the United States and its allies. Such weapons would allow Russia to threaten, with non-nuclear warheads, targets in Europe and eventually the continental United States that, previously, it could only have destroyed with nuclear weapons.

\section{Is China Also Testing and Using Similar Hypersonic Missiles?}

China, like Russia, is developing boost-glide weapons and hypersonic cruise missiles—but let us focus on the boost-glide part of China's program. China is developing a glider that is named WU-14 by the Pentagon and, it is been reported, DF-ZF by China [13]. This glider has been tested repeatedly—at least seven times-over a range of up to 2,000 kilometers, which makes its range substantially shorter than the U.S. advanced hypersonic weapon. It is not clear whether it will be armed with a nuclear warhead, a non-nuclear warhead, or could accommodate either. On balance, it is likely, that in the first instance, it will be armed with nuclear warheads (though the evidence is far from conclusive). Perhaps, over time, China will subsequently develop a non-nuclear-armed glider.

Very much like Russia, China already has the ability to attack U.S. and allied targets with nuclear weapons, so Chinese nuclear-armed boost-glide weapons would merely serve to reinforce the status quo. By contrast, if China develops non-nuclear boost-glide weapons, especially if those could hit the continental United States, it would present the United States with a new and very real technical and military challenge.

\section{How Could the U.S. Defend against Hypersonic Weapons?}

Currently we do not have any effective means of detection and defense mechanisms or for that matter counter-measures against hypersonic weapons because of the way they fly, i.e., they are maneuverable and fly at an altitude that our current defense systems are not designed to operate. For example, an ICBM missile has a predictable trajectory. One can obtain enough information via a remote sensing platform such as the Defense Support Program (DSP) that target acquisition radars can engage in order to intercept a ballistic object.

A ballistic missile is like a fly ball in baseball, the outfielder knows exactly where to catch it because its path is determined by momentum and gravity.

Since hypersonic weapons are maneuverable and unpredictable, therefore, they are difficult to defend against. However, there are potential ways to address hypersonic weapons, but they will be very expensive.

As an example, the Missile Defense Agency is proposing developing a space-based sensor system that would be able to track hypersonic glide vehicles globally, this would be one of the first steps in defending against these new missiles. Or to have powerful radar detection that can dwell on a target during its long trajectory from apogee to target. These types of radar need to detect such weapons way beyond the range of traditional radar systems that are in operation by the militaries around the world today. Their operating range is around a couple hundred miles within the line of sight from the radar source to the target of interest.

However, the traditional detection radar beyond few hundred miles has its own deficiency beyond the line of sight due to the nature of geodesic path that they have to propagate their electromagnetic wave.

However, a new version of detection radar that is driven by an HPM source can reach out further, but it has its own drawbacks.

The problem of microwave breakdown near antennas at high altitudes must be considered in order to find out what the limitation on transmission conditions is [14].

Electrical breakdown is often associated with the failure of solid or liquid insulating materials used inside high voltage transformers or capacitors in the electricity distribution grid, usually resulting in a short circuit or a blown fuse. Electrical breakdown can also 
occur across the insulators that suspend overhead power lines, within underground power cables, or lines arcing to nearby branches of trees.

Airborne radar systems may initiate electrical discharges in front of the antennas at high altitudes because, at ultra-high frequencies, the electric field required to break down air at low pressures is, in general, much less than that required at atmospheric pressure. The processes which determine ultra high frequency (UHF) breakdown have been discovered and verified during the past decade. These have been applied to determining optimum transmission conditions for high flying radar as an example.

Breakdown takes place when an electric field is applied to a gas, and the free electrons move in the direction of the field, constituting a current. There are always a small number of electrons present in any collection of gas because of ionization by cosmic rays or some other phenomenon, such as the photoelectric effect. If the electric field is gradually increased from zero, the gas first appears to obey Ohm's law until the field becomes large enough to impart sufficient energy to some of the electrons to produce secondary electrons by collision. If the electric field is sufficiently great so that many secondary electrons are produced, there will come a point at which the gas will become highly conducting. For a very minute change in voltage or field near this value, the electron concentration and current will change by many orders of magnitude, and the gas will start to glow as it can be seen in Fig. 9 [9].

Electrical breakdown occurs within a gas when the dielectric strength of the gas is exceeded. Regions of intense voltage gradients can cause nearby gas to partially ionize and begin conducting. This is done deliberately in low pressure discharges such as in fluorescent lights. The voltage that leads to electrical breakdown of a gas is approximated by Paschen's Law [15].

It is often asserted that it is impossible to defend against hypersonic weapons because they go too fast. That is empirically not true. The United States has

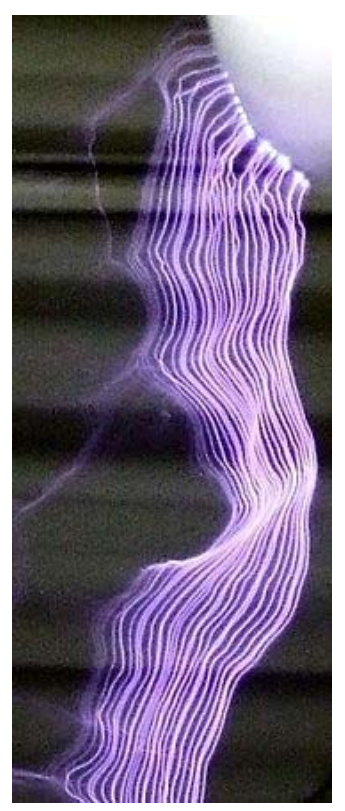

Fig. 9 Electrical breakdown driving electric discharge.

already developed fairly effective "point defenses"-like Patriot and Terminal High-Altitude Area Defense (THAAD) — that can defend small areas against ballistic missiles, which are actually moving faster than hypersonic weapons. (We do not normally class ballistic missiles as a type of hypersonic weapon because they have no ability to maneuver). So, speed, in and of itself, is not an insuperable barrier for missile defenses. Those point-defense systems, and particularly THAAD, could very plausibly be adapted to deal with hypersonic missiles. The disadvantage of those systems is that they can only defend small areas. To defend the whole of the continental United States, you would need an unaffordable number of THAAD batteries. The United States has deployed one missile defense system, the Ground-Based Midcourse System, which is designed to try to defend the whole of the United States against ballistic missiles [16]. For a variety of technical reasons, however, using these "area defenses" to deal with hypersonic weapons is more or less impossible.

Therefore, it is a nuanced picture when it comes to defenses-you can probably defend small areas fairly effectively against gliders, but it is likely to be much more challenging to defend large areas. 
In contrast, a new technology and technical approach looking at a scalar wave (SW) driving an energy wave in longitudinal mode has been proposed by the first author here Zohuri [9]. This approach seems very reasonable and makes sense at least in theory. A small amount of funding needs to be secured to take the theoretical concept into the experimental stage and make a prototype of apparatus that are driven based on the technology known as a SLW [10]. Until such experiments occur, the actual capabilities are only speculative.

\section{What Is the Scalar Wave?}

As it has been understood, the SLW or simply SW does not have the characteristic of electromagnetic (EM) wave and does not behave like EM. However as it is understood from classical electrodynamic (CED) that EM wave has both electric $\vec{E}$ and magnetic fields and power flow in EM wave is driven by means of the Poynting vector, as it is presented by Eq. (1) in the following form as:

$$
\vec{S}=\vec{E} \times \vec{B} \text { Watts } / \mathrm{m}^{2}
$$

Analyzing Eq. (1) indicates that the energy per second crossing a unit area that is normal is oriented in the direction of $\vec{S}$ per definition of two vectors cross-product and it is flowing in EM form.

On the other-hand, an SW has no time varying $\vec{B}$ field. And in some cases, it also has no $\vec{E}$ field. Thus, it has no energy propagated in the EM wave mode as TEM form and shape. Furthermore, with some authors in field of electromagnetic physics, based on non-classical effects of electrodynamics or quantum electrodynamic (QED), they often speak of EM waves not being based on oscillations of electric and magnetic fields.

"For example, it is claimed that there is an effect of such waves on biological systems and the human body. Even medical devices are sold which are assumed to work on the principle of transmitting any kind of information via 'waves' which have a positive effect on human health. In all cases, the explanation of these effects is speculative, and even the transmission mechanism remains unclear because there is no sound theory on such waves, often subsumed under the notion 'scalar waves'” [9].

Additionally, it must be recognized, however, that any vector could be added that could integrate to zero over a closed surface and the Poynting theorem still applies. Thus, there is some ambiguity in even stating the form of Eq. (1), which is the total EM energy flow.

In order to establish the longitudinal potential waves, we develop our theory of EM waves with vanishing field vectors, which is taking place in a "vacuum state". This state also plays a role in the microscopic interaction with matter, so we can restrict our consideration to classical electrodynamics in order to understand better. With $\vec{E}$ and $\vec{B}$ parameters designating the classical electric and magnetic field vectors, then in vacuum they can be written as:

$$
\vec{E}=0
$$

and

$$
\vec{B}=0
$$

Then, the only possibility to find electromagnetic effects is by the potentials. These are defined as vector (magnetic) potential $\vec{A}$ and scalar (electric) potential $\phi$ constituting the "force" fields $\vec{E}$ and $\vec{B}$, and both can be derives as follows.

As we know, Maxell's electromagnetic wave propagation in linear media (i.e. Matter) namely Eq. (9) through Eq. (12) (i.e. their empirical basis with Eq. (9) plus three other Eq. (10) through Eq. (12)) in case of transverse waves, for which the field pointers oscillate perpendicular to the direction of propagation with which we are already familiar with, namely:

$$
\begin{aligned}
& \nabla \times \vec{H}=\vec{J}+\frac{\partial \vec{D}}{\partial t} \\
& \nabla \times \vec{E}=-\frac{\partial \vec{B}}{\partial t}
\end{aligned}
$$




$$
\begin{aligned}
& \nabla \cdot \vec{D}=\rho \\
& \nabla \cdot \vec{B}=0
\end{aligned}
$$

In a homogeneous and isotropic with current density $\vec{J}=0$ as well as $\vec{B}=\mu \vec{H}$ where $\vec{H}$ is the magnetic field intensity and $\vec{D}=\varepsilon \vec{E}$, where $\vec{D}$ is referring to the electric displacement. In these relations, the following definitions are also applied as:

$\varepsilon=$ electric permittivity of the medium

$\mu=$ magnetic permeability of the medium

With speed of light being defined as $c$, then it can be written as $\mu \varepsilon=1 / c^{2}$.

Given the above conditions and definitions, Eq. (4) can be written as a new form as:

$$
\frac{1}{\mu} \nabla \times \vec{B}=\varepsilon \frac{\partial \vec{E}}{\partial t}
$$

Since the magnetic induction has zero divergence it may always be represented as the curl of a vector potential from EM point of view and can be written as:

$$
\vec{B}=\nabla \times \vec{A}
$$

Using Eq. (14) for expression of $\vec{B}$ in Eq. (10) of Maxwell's equation we obtain the following result as:

$$
\begin{aligned}
& \nabla \times \vec{E}=-\frac{\partial}{\partial t}(\nabla \times \vec{A}) \\
& \nabla \times \vec{E}+\frac{\partial}{\partial t} \nabla \times \vec{A}=0
\end{aligned}
$$

Assuming sufficient continuity of the fields to interchange the spatial and temporal differentiations, this can be written as:

$$
\nabla \times\left[\vec{E}+\frac{\partial \vec{A}}{\partial t}\right]=0
$$

The vector $\vec{E}+\partial \vec{A} / \partial t$ thus has zero curl and can be written as the gradient of a scalar as:

$$
\vec{E}=-\nabla \phi-\frac{\partial \vec{A}}{\partial t}
$$

In this case $\phi$ is the scalar (electric) potential and $\vec{A}$ is the (magnetic) vector potential in Eqs. (14) and (17).

These potentials satisfy wave equations which are very similar to those satisfied fields. The wave equation for $\vec{A}$ is derived by substituting the expressions given in Eqs. (9) and (12) for $\vec{B}$ and $\vec{E}$ into Eq. (13), with result:

$$
\begin{aligned}
& \frac{1}{\mu} \nabla \times(\nabla \times \vec{A})=\varepsilon \frac{\partial}{\partial t}\left\{-\nabla \phi-\frac{\partial \vec{A}}{\partial t}\right\} \\
& \frac{1}{\mu} \nabla \times \nabla \times \vec{A}+\varepsilon \frac{\partial}{\partial t}\left\{-\nabla \phi-\frac{\partial \vec{A}}{\partial t}\right\}=0
\end{aligned}
$$

Using vector identity $\nabla \nabla \cdot-\nabla^{2}$ for $\nabla \times \nabla \times$ and multiplying by $\mu$ in second form of Eq. (18), and using $\mu \varepsilon=1 / c^{2}$, we obtain the following result as:

$$
-\nabla^{2} \vec{A}+\frac{1}{c^{2}} \frac{\partial^{2} A}{\partial t^{2}}+\nabla \nabla \cdot \vec{A}+\frac{1}{c^{2}} \nabla \frac{\partial \phi}{\partial t}=0
$$

Eq. (19) takes place under vacuum conditions or homogeneous media where current density $\vec{J}=0$.

Until now only the curl of vector potential $\vec{A}$ has been specified; the choice of the divergence of $\vec{A}$ is still arbitrary. It is clear from Eq. (19) imposing the so-called Lorentz gauge condition, where:

$$
\nabla \cdot \vec{A}+\frac{1}{c^{2}} \frac{\partial \phi}{\partial t}=0
$$

Results are in a considerable simplification. If this condition is satisfied, then $\vec{A}$ satisfies the wave equation as:

$$
\nabla^{2} \vec{A}-\frac{1}{c^{2}} \frac{\partial^{2} \vec{A}}{\partial t^{2}}=0
$$

(Vector potential wave)

Furthermore, using Eq. (17) in Eq. (11) for vacuum or homogeneous media $\rho=0$ with $\vec{D}=\varepsilon \vec{E}$, we obtain:

$$
-\varepsilon\left[\nabla \cdot \nabla \phi+\nabla \cdot \frac{\partial \vec{A}}{\partial t}\right]=0
$$


Interchanging the order of the divergence and the time derivative operating on $\vec{A}$ and using the Lorentz condition Eq. (20) everything leads to:

$$
\nabla^{2} \phi-\frac{1}{c^{2}} \frac{\partial^{2} \phi}{\partial t^{2}}=0
$$

(Scalar potential wave)

Thus, by imposing the Lorentz condition, both the scalar and vector potentials are forced to satisfy inhomogeneous wave equations of similar forms. However, the problem of finding the general solution of the inhomogeneous scalar wave equation is analogous to finding the general solution of Poisson's equation.

However, a solution appears to exist for the special case of $\vec{E}=0, \vec{B}=0$, and $\nabla \times \vec{A}=0$, for a new wave satisfying:

$$
\begin{gathered}
\vec{A}=\nabla S \\
\phi=-\frac{1}{c^{2}} \frac{\partial S}{\partial t}
\end{gathered}
$$

$S$ then satisfies:

$$
\nabla^{2} S-\frac{1}{c^{2}} \frac{\partial^{2} S}{\partial t^{2}}=0
$$

Mathematically $S$ is a "potential" with a wave equation, one that suggests propagation of this wave even through $\vec{E}=\vec{B}=0$ and the Poynting theorem indicates no EM power flow.

From Eq. (6) and condition of establishing it in above, there is the suggestion of a solution to Maxwell's equations involving a scalar wave with potential $S$ that can propagate without Poynting vector EM power flow. But the question then arises as to where the energy is drawn from to sustain such a flow of energy.

A vector that integrates to zero over a closed surface might be added in the theory, as suggested in beginning of Section 9 of book by Zohuri [9]. Another is the possibility of drawing energy from the vacuum, assuming net energy could be drawn from "free space". As is suggested by quantum mechanics (QM) it allows random energy in free space but conventional or classical electromagnetics (CEM) theory has not allowed this to date.

Random energy in free space that is built of forces fields that sum to zero is a possible approach. If so, these might be a source of energy to drive the $S$ wave drawn from "free space".

Note that with condition of $\vec{E}=\vec{B}=0$, both Eqs. (14) and (17) will reduce to:

$$
\nabla \phi=-\frac{\partial \vec{A}}{\partial t}
$$

and

$$
\nabla \times \vec{A}=0
$$

From Eq. (26), one follows immediately that the vector potential $\vec{A}$ is vortex free, representing a laminar flow. The gradient of the scalar potential is coupled to the time derivative of the vector potential so both are not independent of one another [9].

The SW could be accompanied by a vector potential $\vec{A}$.

An SW is a non-linear, non-Hertzian, standing wave capable of supporting significant effects including carrying information and inducing higher levels of cellular energy.

Scalar waves can be created by wrapping electrical wires around a figure eight in the shape of a Möbius coil. When an electric current flows through the wires in opposite directions, the opposing EM fields from the two wires cancel each other and create a scalar wave.

The scalar waves cannot be detected directly because they do not impart energy and momentum to matter. On the other hand, they impart phase shifts to matter, and they may be detected through interference means. Because of their elusive nature they may also be called scalar vacuum waves. The underlying scalar field is already known to physicists in the context of quantum field theory and is known as the scalar gauge field. It should be noted at this time, that other researchers have reported the observation of fields 
which behave qualitatively similar to the predicted scalar fields. The extension of the forceless field concept to the nucleonic field should yield higher order fields with even more interesting properties than the scalar fields. This matter is under investigation.

The SLW, does not damp out with $1 / r^{2}$ dependence, where $r$ is distance between the source of the SLW and its target. The dispersion of such a wave far from the source is correlated to $1 / r$ as is shown in Eq. (28) with strength source $S_{0}$.

$$
S=\frac{S_{0}}{r} e^{j(\omega t-k r)}
$$

The power per unit area of the wave front is proportional to the square of the strength $S$ of the wave. However, the total power must be independent of distance from the source. The area of the spherical surface over which the wave is spread at a distance $r$ from the source is $r^{2}$ in the case of the near field. Hence, $S^{2} r^{2}$ is constant, but far from the source (i.e. far field), the strength $S$ of such a wave is described by Eq. (22) which is an exact solution of the linear SW equation.

$$
\left\{\begin{array}{l}
S=\frac{S_{0}}{r}\left\{e^{-j k[r-(a / 2) \cos \theta]}-e^{-j k[r+(a / 2) \cos \theta]}\right\} \\
S=\frac{2 j S_{0}}{r}\left(e^{-j k r}\right) \sin [(k a / 2) \cos \theta]
\end{array}\right.
$$

Eq. (29) is derived based on Fig. 10 with some mathematical manipulation as well, assuming Eq. (1) holds exactly and that the source to the left is equal and opposite to the source to the right (i.e. two sources of scalar waves dueling on target, as depicted in Fig. 10).

The strengths of the two waves are not exactly equal and opposite at the point $P$ for two reasons:

(1) The right-hand source is nearer to $P$ and hence the wave from this source has a slightly greater strength than the wave from the source to the left;

(2) Because the two sources are at slightly different distances, the waves from them are not exactly $180^{\circ}$ out of phase at $P$.

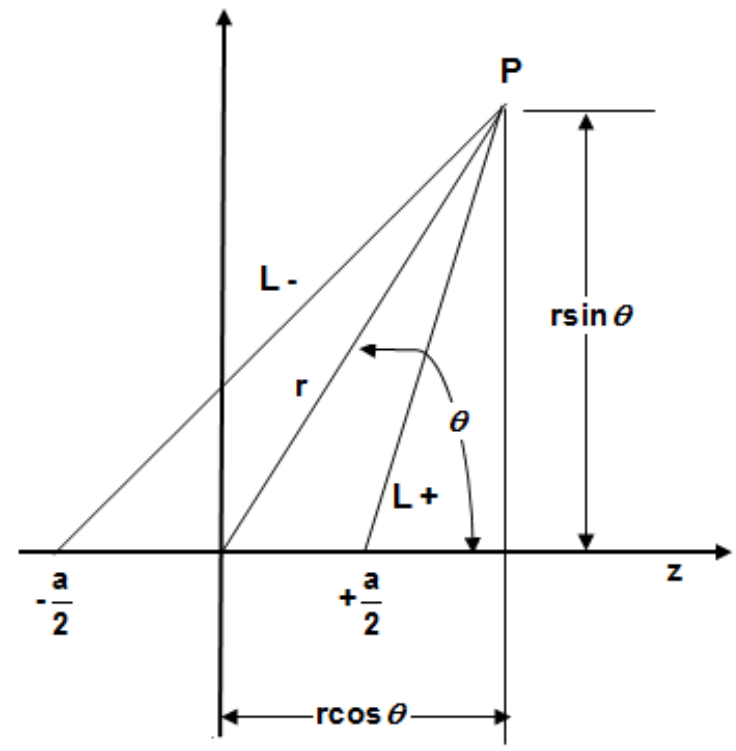

Fig. 10 Two different sources of wave.

NORMAL IONOSPHERIC REFLECTING LAYERS $(100-300 \mathrm{~km})$

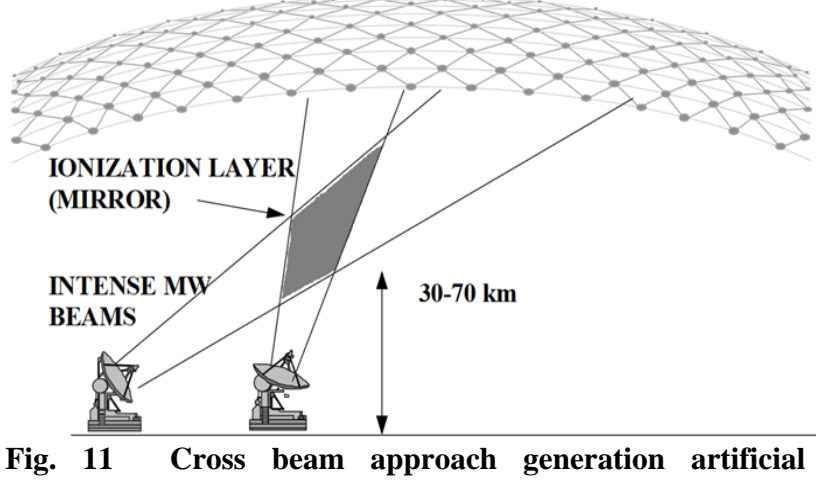
ionospheric mirror.

In this paper we do not show details of analysis that get to the result in Eq. (2), however the signals from the two sources may arrive at point $P$ in phase, so that the strengths add, or $180^{\circ}$ out of phase, so that the strengths cancel.

\section{Transmitters and Receivers for SLW}

Horst Eckardt [17] in his paper suggests the following procedure for transmitting and receiving SLW, where a sender for longitudinal potential waves has to be a device which avoids producing $E$ and $B$ fields but sends out oscillating potentials. He discusses two propositions how this can be achieved technically. In the first case, we use two ordinary transmitter 
antennas (with directional characteristic) with distance of half a wavelength (or an odd number of half waves). This means that ordinary electromagnetic waves cancel out, assumed that the near field is not disturbing significantly. Since the radiated energy cannot disappear, it must propagate in space and is transmitted in form of potential waves. This is depicted in Fig. 12.

A more common example is a bifilar flat coil, for example, from the patent of Tesla [11] (see Fig. 13), second drawing. The currents in opposite directions affect a nihilation of the magnetic field component, while an electric part may remain due to the static field of the wires.

Construction of a receiver is not so straightforward. In principle no magnetic field can be retrieved directly from $\vec{A}$ due to Eq. (27). The only way is to obtain an electrical signal by separating both contributing parts in Eq. (20) so that the equality of Eq. (17) is out weighted, and an effective electric field remains which can be detected by conventional devices. A very simple method would be to place two plates of a capacitor in distance of half a wavelength (or odd multiples of it). Then the voltage in space should have an effect on the charge carriers in the plates, leading to the same effect as if a voltage had been applied between the plates. The real voltage in the plates or the compensating current can be measured (Fig. 14).

The "tension of space" operates directly on the charge carriers while no electric field is induced. The $\partial \vec{A} / \partial t$ part is not contributing because the direction of

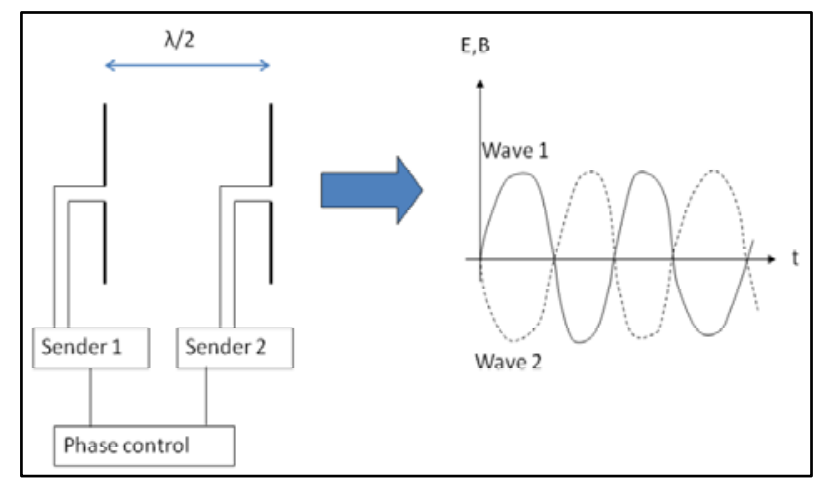

Fig. 12 Suggestion for a transmitter of longitudinal potential waves.
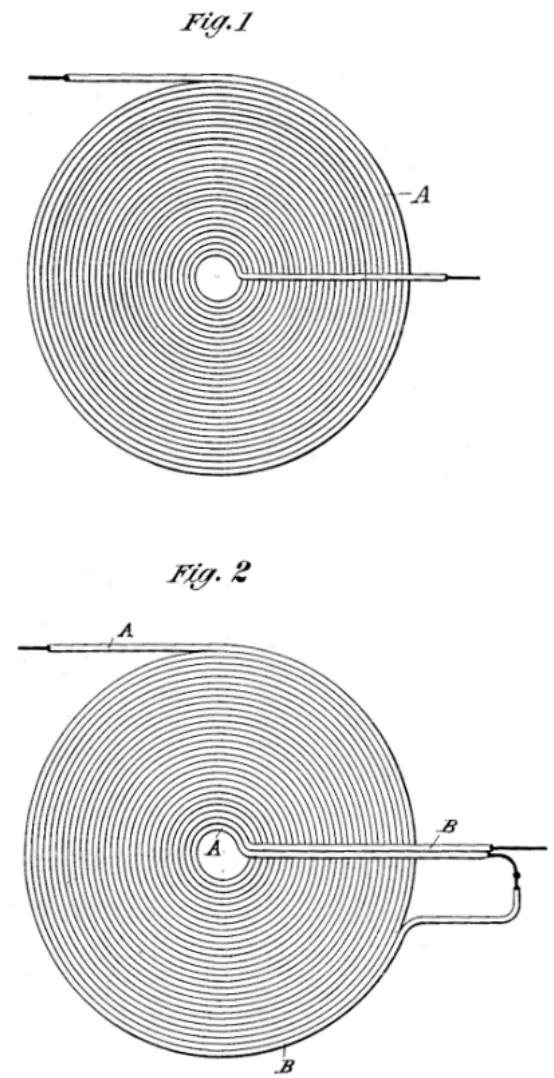

Fig. 13 Tesla coils according to the patent of his [11].

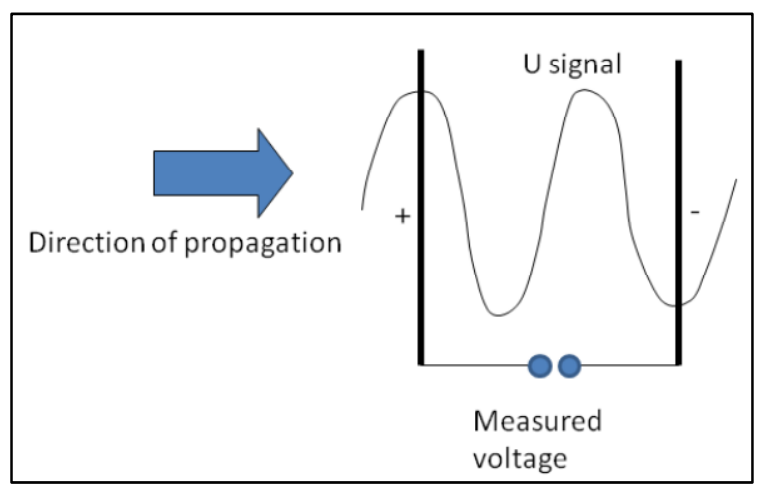

Fig. 14 Suggestion for a receiver of longitudinal potential waves (capacitor) [17].

the plates is perpendicular to it, i.e. no significant current can.

Another possibility of a receiver is to use a screened box (Faraday cage). If the mechanism described for the capacitor plates is valid, the electrical voltage part of the wave creates charge effects which are compensated immediately due to the high conductivity of the material. As is well known, the interior of a Faraday cage is free of electric fields. The potential is constant 


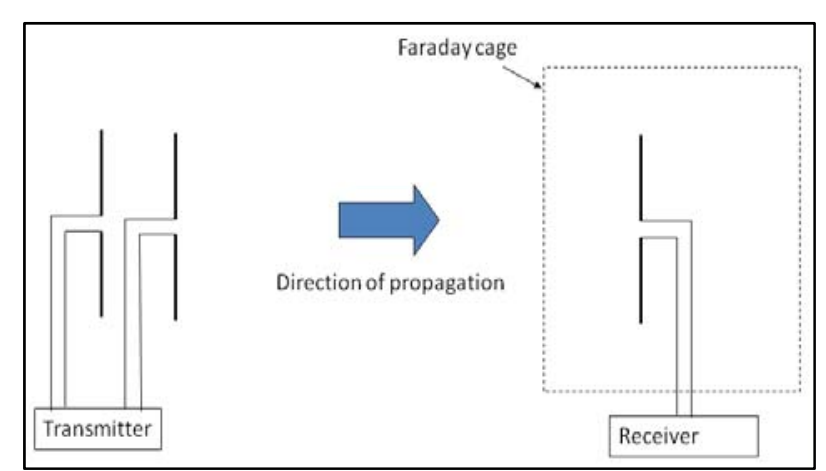

Fig. 15 Suggestion for a receiver of longitudinal potential waves (Faraday cage) [17].

because it is constant on the box surface. Therefore, only the magnetic part of the wave propagates in the interior where it can be detected by a conventional receiver (see Fig. 15).

Another method of detection is using vector potential effects in crystalline solids. As is well known from solid state physics, the vector potential produces excitations within the quantum mechanical electronic structure, provided the frequency is near to the optical range. Crystal batteries work in this way. They can be engineered through chemical vapor deposition of carbon. In the process you get strong light weight crystalline shapes that can handle lots of heat and stress (by high currents). For detecting longitudinal waves, the excitation of the electronic system has to be measured, for example by photoemission or other energetic processes in the crystal.

All these are suggestions for experiments with longitudinal waves. Additional experiments can be performed for testing the relation between wave vector $k$ which is defined from the wave length $\lambda=2 \pi / k$ and frequency $\omega$ to check if this type of waves propagates with ordinary velocity of light $C$, see Ref. [17].

$$
c=\frac{\omega}{k}
$$

As pointed out by H. Eckardt, D. W. Lindstrom [18], the speed of propagation depends on the form of the waves and possibly could be a non-linear step function as well. The experimental setup is illustrated in Fig. 10 and it can directly be used as for finding the $\omega(k)$ relation due to the wavelength and frequency that could be measured at the same time and analysis can be seen from the solution to Eq. (16), where as a simple example we assume a sine-like behavior of vector potential $\vec{A}$ in direction of x-axis with direction $\vec{k}$ wave vector, space coordinate vector $\vec{x}$ and time frequency $\omega$ as:

$$
\vec{A}=A_{0} \sin (\vec{k} \cdot \vec{x}-\omega t)
$$

Substituting this solution into Eq. (20), we obtain:

$$
\frac{\partial \phi}{\partial t}=\nabla \phi=A_{0} \omega \cos (\vec{k} \cdot \vec{x}-\omega t)
$$

This condition has to be met for any potential $\phi$. We make the following approach as:

$$
\phi=\phi_{0} \sin (\vec{k} \cdot \vec{x}-\omega t)
$$

in order to find

$$
\frac{\partial \phi}{\partial t}=\nabla \phi=k \phi_{0} \cos (\vec{k} \cdot \vec{x}-\omega t)
$$

Comparing Eqs. (34) with (32), we can see that the constant $A_{0}$ can be defined as:

$$
A_{0}=k \frac{\phi_{0}}{\omega}
$$

It is obvious that the waves of $\vec{A}$ and $\phi$ have the same phases, and naturally with these results we can now consider the energy density of such a combined wave, and generally speaking it can be given as:

$$
\mathcal{E}=\frac{1}{2} \varepsilon_{0} \vec{E}^{2}+\frac{1}{2 \mu_{0}} \vec{B}^{2}
$$

From Eqs. (20) and (21), we can observe that the magnetic field disappears identically, but the electric field is a vanishing sum of two terms which are different from zero [9].

These two terms evoke an energy density $\mathcal{E}$ of space where the wave propagates. This can not be obtained out of the force fields (these are zero) but must be computed from the constituting potentials. As discussed we have to write:

$$
\mathcal{E}=\frac{1}{2} \varepsilon_{0}\left(\dot{\vec{A}}^{2}+(\nabla \phi)^{2}\right)
$$


With help from Eqs. (31) and (32) it follows that:

$$
\mathcal{E}=\varepsilon_{0} k^{2} \phi_{0}^{2} \cos ^{2}(\vec{k} \cdot \vec{x}-\omega t)
$$

This is an oscillating function, meaning that the energy density varies over space and time in phase with the propagation of the wave. All quantities are depicted in Fig. 10. Energy density is maximal where the potentials cross the zero axis. There is a phase shift of 90 degrees between both.

Further, analyses of Fig. 15 indicate that, there is an analogy between longitudinal potential waves and acoustic waves.

It is well known that acoustic waves in air or solids are mainly longitudinal too. The elongation of molecules is in direction of wave propagation as shown in Fig. 16. This is a variation in velocity. Therefore, the magnetic vector potential can be compared with a velocity field. The differences of elongation evoke a local pressure difference. Where the molecules are pressed together, the pressure is enhanced, and vice versa.

From conservation of momentum, the force $\vec{F}$ in a compressible fluid is:

$$
\vec{F}=\frac{\partial u}{\partial t}+\frac{\Delta p}{\rho}
$$

where, $u$ is the velocity field, $p$ is the pressure and $\rho$ is the density of the medium.

This is in full analogy to Eq. (12). In particular we see that in the electromagnetic case spacetime must be "compressible", otherwise there was no gradient of the scalar potential. As a consequence, space itself must be compressible, leading us to the principles of general relativity (see Fig. 17).

\section{Scalar Waves as Weapons}

Since the SW carries no energy in its beam, it can only manipulate the energy available in any scenario. The object of targeting an SW at a hypersonic vehicle is to manipulate the plasma impinging on the surface of the vehicle. The SW may amplify or attenuate the

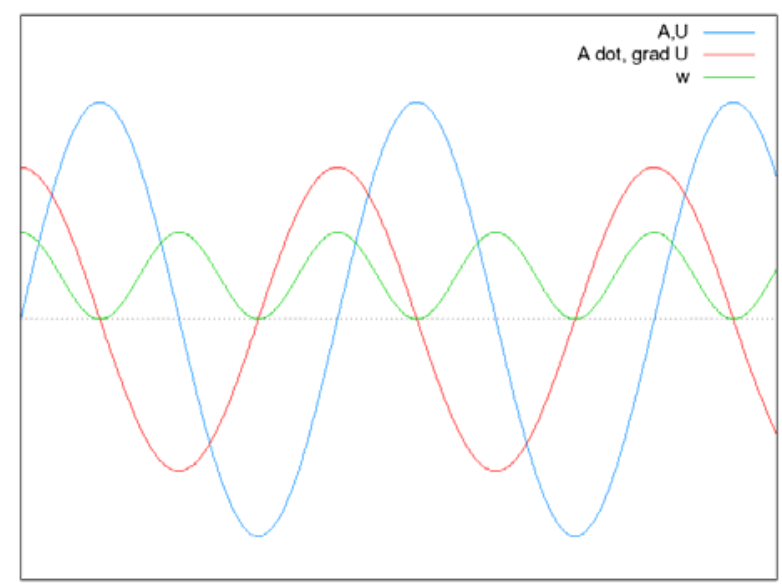

Fig. 16 Phases of potential $\vec{A}$ and $\phi$, and energy density $\mathcal{E}$.

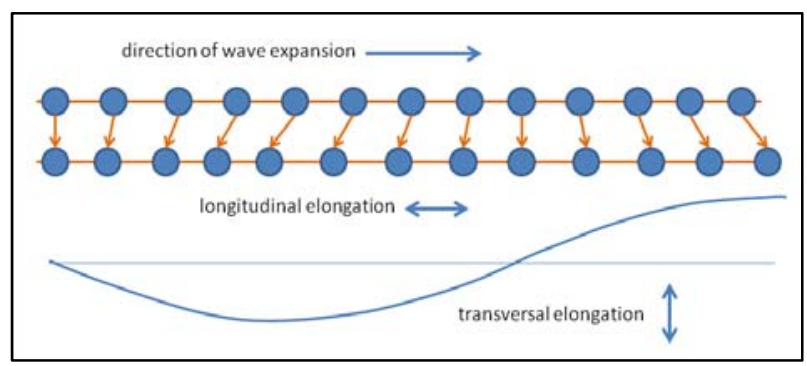

Fig. 17 Schematic representation of longitudinal and transversal waves.

forces on the surface of the vehicle in such a way that the structural integrity of the vehicle is compromised. Or it may perturb the plasma in such a way that the vehicle is driven off target or malfunctions. Because the SW travels at the speed of light and oscillates at megahertz frequencies, the hypervelocity technology is a significant advantage. However, the plasma generated by the hypervelocity vehicle is required to give the SW something to manipulate.

\section{Scalar Waves Superweapon Conspiracy Theory}

According to Tom Bearden [19], the scalar interferometer is a powerful superweapon that the Soviet Union used for years to modify weather in the rest of the world. It taps the quantum vacuum energy, using a method discovered by T. Henry Moray in the 1920s. However, some conspiracy theorists believe Bearden is an agent of disinformation on this topic, 
thus we leave this matter to the reader to make their own conclusions and be able to follow up their own findings and this paper does not claim any of these matters are false or true.

However, in the 1930's Tesla announced other bizarre and terrible weapons: a death ray, a weapon to destroy hundreds or even thousands of aircraft at hundreds of miles range, and his ultimate weapon to end all war-the Tesla shield, which nothing could penetrate. However, by this time no one any longer paid any real attention to the forgotten great genius. Tesla died in 1943 without ever revealing the secret of these great weapons and inventions. Tesla called this super weapon a scalar potential howitzer or death ray as artistically depicted in Fig. 18. Such a weapon apparently was demonstrated by Soviets at their Saryshagan missile range during the peak of the strategic defense initiative (SDI) time period. It was mentioned during strategic arms limitation talks (SALT) treaty negotiations.

According to Bearden, in 1981 the Soviet Union had discovered and weaponized the Tesla SW effects. Brezhnev undoubtedly was referring to it in 1975 when the Soviet side at the SALT suddenly suggested limiting the development of new weapons "more frightening than the mind of man had imagined”. One of these weapons is the Tesla howitzer recently completed at Saryshagan ballistic missile range near the Sino-Soviet border in Southern Russia, according to a high-level U.S. official and presently considered to be either a high-energy laser or a particle beam weapon, (see Aviation Week \& Space Technology, July 28, 1980, p. 48 for an artistic conception). A retouched photograph of the Saryshagan installation extracted from Aviation Week is shown as Fig. 19.

Bearden claims that, the Saryshagan howitzer actually is a huge Tesla scalar interferometer with four modes of operation. One continuous mode is the Tesla shield, which places a thin, impenetrable hemispherical shell of energy over a large defended area. The 3-dimensional shell is created by interfering two

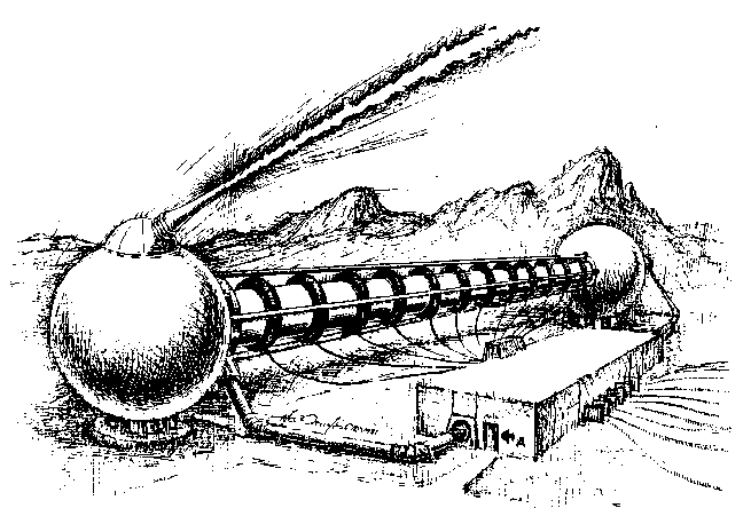

Fig. 18 Scalar Potential Interferometer.

Multimode Tesla Weapon.

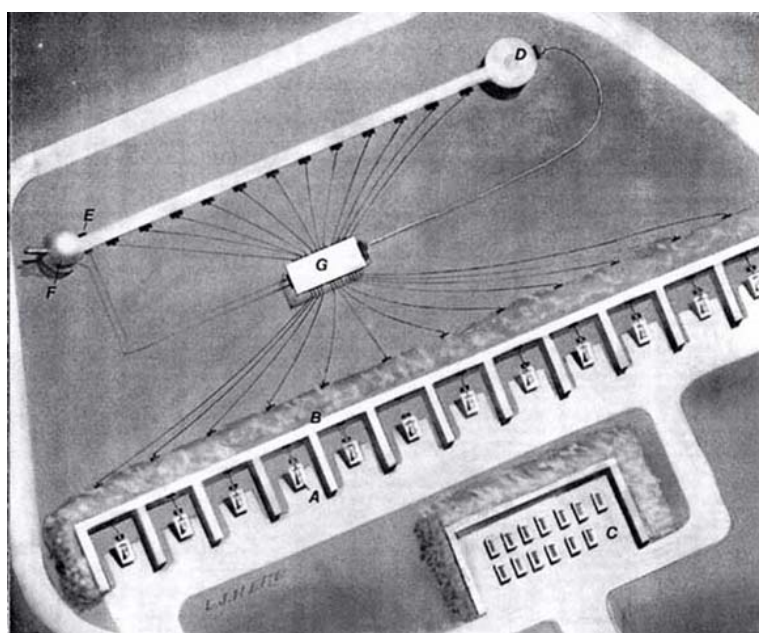

Fig. 19 Aviation Week \& Space Technology July 28, 1980, p. 48.

Source: the photo is taken by U.S. High-Resolution Reconnaissance Satellite KH-11.

Fourier-expansion, 3-dimensional scalar hemispherical patterns in space so they pair-couple into a dome-like shell of intense, ordinary electromagnetic energy. The air molecules and atoms in the shell are totally ionized and thus highly excited, giving off intense, glowing light. Anything physical which hits the shell receives an enormous discharge of electrical energy and is instantly vaporized-it goes pfft! Like a bug hitting one of the electrical bug killers now so much in vogue (see Fig. 15).

Bearden goes on further to say that, if several of these hemispherical shells are concentrically stacked, even the gamma radiation and elector-magnetic pulse (EMP) from a high altitude nuclear explosion above the stack can not penetrate all the shells due to 
repetitive absorption and reradiation and scattering in the layered plasmas.

Bearden speculates about many other effects of scalar waves [19].

\section{Disclaimer}

All of the information contained in this paper was obtained from open sources. The opinions expressed are the authors alone.

\section{References}

[1] The Diplomat. http://thediplomat.com/2018/12.

[2] Pai, S. 1962. Magnetogasdynamics and Plasma Dynamics. Vienna: Springer-Verlag. Englewood Cliffs, NJ: Prentice-Hall, Inc.

[3] Rosa, R. J. 1968. Magnetohydrodynamic Energy Conservation. NY: McGraw-Hill Book Company.

[4] Gurijanov, E. P., and Harsha, P. T. 1996. "AJAX: New Directions in Hypersonic Technology.” Presented at Seventh Aerospace Planes and Hypersonic Technology Conference, Norfolk, VA.

[5] Bruno, C., Czysz, P. A., and Murthy, S. N. B. 1997. "Electro-Magnetic Interactions in a Hypersonic Propulsion System.” Presented at 33rd AIAA/ASME/SAE/ASEE Joint Propulsion Conference, Seattle, WA.

[6] Litchford, R. J., Cole, J. W., Bityurin, V. A., and Lineberry, J. T. 2000. "Thermodynamic Cycle Analysis of Magnetohydrodynamic-Bypass Hypersonic Airbreathing Engines.” Journal of Propulsion and Power 17 (2).
[7] Murthy, S. N. B., and Blankson, I. M. 2000. "MHD Energy Bypass for Turbojet-Based Engines.” Presented at 51st International Astronautical Congress, Rio de Janeiro, Brazil.

[8] Macheret, S. O., Shneider, M. N., and Miles, R. B. 2004. "Magnetohydrodynamic and Electrohydrodynamic Control of Hypersonic Flows of Weakly Ionized Plasmas.” AIAA JOURNAL 42 (7).

[9] Zohuri, B. 2018. Scalar Wave Driven Energy Applications. New York: Springer Publishing Company.

[10] Zohuri, B. 2018. "Principle of Scalar Electrodynamics Phenomena Proof and Theoretical Research.” Journal of Energy and Power Engineering 12: 408-17. doi:10.17265/1934-8975/2018.08.005.

[11] Tesla, N. 1894. Coil for electro-magnets. U.S. Patent 512,340 .

[12] The Diplomat. http://thediplomat.com/2018/12.

[13] “DF-ZF.” https://en.wikipedia.org/wiki/DF-ZF.

[14] MacDonald, A. D. 1966. Microwave Breakdown in Gases. New York: Wiley Publisher.

[15] Zohuri, B. 2019. Directed Energy Beam Weapons, the Dawn of New Age Defenses. New York: Springer Publishing Company.

[16] The Diplomat. http://thediplomat.com/2018/12.

[17] Eckardt, H. 2012. "What are 'Scalar Waves'?" www.aias.us, Www.atomicprescision.com, www.upitec.org.

[18] Eckardt, H., Lindstrom, D. W. 2011. "Solution of the ECE Vacuum Equations.” In Generally Covariant Unified Field Theory. Vol. 7, pp. 207-27.

[19] Bearden, T. E. 1981. Solutions to Tesla's Secrets and the Soviet Tesla Weapons. Millbrae, California: Tesla Book Company. 